\title{
Critical aspects for a CFD simulation compared with PIV analysis of the flow field downstream of a prosthetic heart valve
}

\author{
M Grigioni, C Daniele, C Del Gaudio, A Balducci, U Morbiducci, \\ G D'Avenio, V Barbaro \\ Laboratory of Biomedical Engineering, Istituto Superiore di Sanità, \\ Roma, Italy
}

\begin{abstract}
The investigation of the fluid dynamic field determined by the implantation of a medical device is of fundamental importance from a bioengeneering and a clinical point of view: it is well-known that the modification of the physiological fluidic condition could generate thrombogenic and/or hemolytic phenomena. Several techniques can be implemented for this study (PIV, PTV, CFD), but none of them can be regarded as completely exhaustive; intrinsic technical limitations can lead to approximate results. This paper presents a numerical analysis of the flow field downstream of a prosthetic bileaflet heart valve in aortic position, placed in a glass test chamber designed as the natural aortic root for in vitro experiment. PIV analysis was performed as experimental guideline and its results were compared to those obtained by a 2D CFD simulation in order to verify if a simplified numerical model could furnish results sufficiently close to the PIV ones. This comparison was performed for a fraction of the systolic period (where the valve can be considered fully open) assuming a systolic flow of $1 \mathrm{l} / \mathrm{min}$ at $70 \mathrm{bpm}$. The agreement of the results of these two methodologies suggests not only that a numerical model, although simplified, can be helpful as complementary to an experimental analysis (e. g., investigation of areas of the fluid domain where the PIV technique suffers from lack of resolution or illumination), but also indicate critical aspects to improve the numerical model itself and to refine the fluid dynamical assumptions to be implemented. At the same time it could be necessary to define a more accurate approach for CFD and PIV set-ups, being the latter much more related to the experimental condition.
\end{abstract}




\section{Introduction}

The implementation of CFD simulation, combined with experimental technique such as Particle Image Velocimetry (PIV), can be considered as an useful tool, in bioengineering field, for the investigation of the flow domain of medical devices. This point of view relies on the fact that just one methodology can not be sufficient for a complete description of a complex fluidic pattern, in this way the lack of resolution of one technique can be integrated by the results of the second one. The present paper shows a two-dimensional model of a bileaflet valve, similar to a Carbomedics of $19 \mathrm{~mm}$ tissue annulus diameter: the valve was inserted in a geometrical model representing the shape of the aortic root, drawn on the basis of the anatomical findings of other researchers [1]. The need to be closer to the anatomical aortic root shape relies on the fact that the fluid dynamics in this region is strictly correlated to the degree of perfusion. Some authors [2] showed that the presence of the Valsalva sinuses ensure a forward flow in aorta that is greater than the corresponding one in an aorta without sinuses: the backward flow is about $2 \%$ of the forward one in presence of the sinuses while it raises to $25 \%$ in their absence, in the case of a native valves. Furthemore the vortex formation in this district supports the opening and the closing motion of the leaflets valve during the cardiac cycle and helps to ensure a good washout in the diastolic period that prevents the onset of thrombi. A twodimensional CFD analysis of the pulsatile flow through and downstream of the aortic prosthetic bileaflet heart valve during a fraction of the systolic ejection, when the leaflets of the valve are completely open, for a cardiac output of 1 $1 /$ min, was obtained using the computational package FIDAP (Fluent Inc., Lebanon, NH, USA), based on the finite element methodology. The flow velocity regime has been set in order to limit high velocity gradients in the PIV experiments which can determine, e. g., a variation in the seeding density and therefore measurement errors [3]. For the validation of the computational model, the velocity profiles located in the largest section of the aortic root, were compared with the ones obtained by PIV technique performed on a glass blown epitrochoidal-shaped aortic root, placed in the aortic test section of the pulse duplicator of the Sheffield University, for the same cardiac output. This experimental procedure can characterize the flow field, in the light laser plane, of the prosthetic valve by imaging the displacement of the particles seeded into the fluid; in this way it is possible to have $2 \mathrm{D}$ informations from a complete $3 \mathrm{D}$ geometry.

\section{Materials \& Methods}

A 2D model, based on the design of a Carbomedics valve ( $19 \mathrm{~mm}$ tissue-annulus diameter, opening angle respect to the symmetry axis: $12^{\circ}$ ), placed in the aortic test section of a modified Sheffield Pulse Duplicator, was developed. The valve was considered to be in its fully-open position during the time interval of investigation, modelling a specific pulsatile flow testing.

The geometrical model adopted to represent Valsalva sinuses is based on the study performed in [1]. The cross section of the aortic root can be mathematically described by epitrochoids. An epitrochoid is defined as the curve 
described by a point lying inside a circle that rolls on a second one; the shape of the epitrochoid is a function of the ratio of the respective circles diameters and of the parameter $\lambda=\mathrm{CP} / \mathrm{b}$, where CP is the distance between the centre of the rolling circle and the point $(P)$ that describes the epitrochoid and $b$ is the radius of the rolling circle.

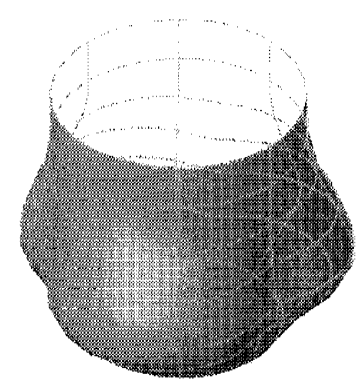

Figure 1 Model of the aortic root surface

It is assumed that the diameters ratio is $3: 1$ because of the anatomical symmetry of $3 \times 120^{\circ}$ (three sinuses) and $\lambda=0.5$ (normal aortic root). For a physiological subject the height of the aortic root is equal to the nominal diameter of the inlet section (as in [1]): reconstructing all the epitrochoidal sections in order to have the aortic root surface, the result is showed in Figure 1. This shape was used to realize three graphite moulds in which a glass tube was blown at National Institute of Health (ISS). The geometrical model implemented for the simulation, comprehending the valve, the left ventricle outflow tract and a straight cylindrical tube, is shown in Figure 2. The model previously described was solved by using the FEM general purpose computational fluid dynamics code FIDAP, based on the Galerkin method of weighted residuals. The mesh-grid has been realized by means of 4 nodes linear blocks. As far as the fluid dynamics model, blood was modelled as an homogeneous, incompressible Newtonian fluid [4-9], with a specific mass $\rho$ of $1055 \mathrm{~kg} / \mathrm{m}^{3}$ and a constant dynamic viscosity $\mu$ of $3.6 \mathrm{mPa} \cdot \mathrm{s}$. Flow has been assumed to be laminar $[4-6,7,9-11]$ and timedependent. The flow motion through the fully open bileaflet mechanical heart valve model up to peak systole, has been described by the principle of momentum conservation, expressed by the Navier-Stokes equations and by the continuity equation, representing the principle of mass conservation. For a transient analysis the flow motion governing equations, in the Cartesian coordinates system described in Figure 2, are given as:

$$
\begin{array}{cl}
\rho(V \bullet \nabla) V=-\nabla p+\mu \nabla^{2} V & \text { (momentum) } \\
\nabla \bullet V=0 & \text { (mass) (2) }
\end{array}
$$

where $V$ and $p$ represent, respectively, the velocity vector and the pressure in each point of the fluid domain. We considered, for the problem under study, impermeable walls, with imposed no slip boundary conditions at the rigid aortic root walls, on the leaflets, ind on the internal surface of the mechanical valve orifice. Systolic flow into the model was plug-shaped, corresponding to a flow rate of $11 / \mathrm{min}$ and to 70 beats per minute. 


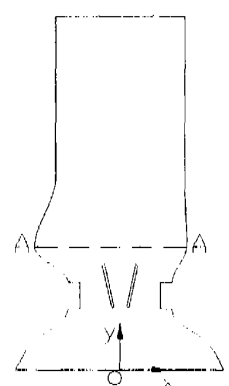

Figure 2: Geometrical model for CFD investigation. In the section AA the comparison of CFD - PIV results is performed

Previous experimental investigations [12] suggested, downstream of bileaflet mechanical valves, laminar and transitional flow regimes for the modeled system under investigation. In the present study, a peak Reynolds number of 3200 was calculated for the model considering the inlet aortic diameter and the velocity at peak systole: as this value of the Reynolds number belongs to the range corresponding to the transition flow region, no turbulence models were invoked for the current investigation considering, as in previous works $[4-6,9]$, that the full solution of the Navier Stokes equations, together with an adequate mesh strategy, should furnish a reasonable prediction of the flow field. The time interval chosen for CFD analysis was located around systolic peak. Previously performed high speed video analysis of the opening of the Carbomedics valve during a pulsatile flow test in the experimental set up reproduced by the model, showed that the leaflets can be considered as fully open $[9,13]$ within $35 \mathrm{~ms}$ during the systole. In order to solve the nonlinear system of matrix equations derived from the FEM discretization of the flow equations on the computational grid, the successive substitutions iterative method has been chosen. The second order trapezoid implicit time integration scheme has been implemented with a fixed time increment. Pulsatile study has been performed in a mock circulatory loop making use of the properly modified Sheffield pulse duplicator [14]. Test fluid was a water - glycerine solution about $33 \%$ and seeding particles were a basic anion exchanger $(50-150 \mu \mathrm{m}$ diameter $)$. Once set the pulsatile flow conditions at $70 \mathrm{bpm}$, according to [15], the cardiac output of $1 / / \mathrm{min}$, with a mean aortic pressure of $100 \mathrm{mmHg}$, have been investigated. Characteristic nondimensional numbers related to these setting parameters were $\operatorname{Re}=3200$ (Reynolds number) and $\mathrm{Wo}_{\mathrm{O}}=10.6$ (Womersley number), calculated considering $\mathrm{U}=0.626 \mathrm{~m} / \mathrm{s}$ (maximum instantaneous velocity), $\mathrm{v}=3.7 \mathrm{cSt}$ (blood kinematic viscosity) and $\mathrm{D}=19 \mathrm{~mm}$. The command waveform for the pulse duplicator has been set to attain a $35 \%$ systole/cycle ratio. The image acquiring and the system of lighting for PIV investigation were done by means of the Kodak Ektapro high speed video camera (1000 frame/s) and of the Laser Innova 70C Nd-YAG (3W, $514.5 \mathrm{~nm}$ ). Each velocity field was calculated using a commercial PIV software (AEA Visiflow) (cross-corralation of two consecutive frames) setting the Interrogation Box (IB) to $32 \times 32$ pixels dimensions with about $70 \%$ overlapping. 


\section{Results}

Contour plot of the axial component of velocity in the flow domain under investigation, corresponding to the peak flow $(t=0.165 \mathrm{~s})$, is shown in figure 3 , illustrating the CFD model prediction on the plane under investigation; the onset of a strong vortex is clearly predicted in the sinus area at that time. Figure 4 shows the PIV results at the maximum flow rate: also from this figure it is possible to notice the formation of a recirculating region within the sinus district. Moreover, the fluid passing through the lateral orifices increases its velocity more than that flowing through the central one, because of the larger size of these areas; this situation remains the same until the beginning of the decelaration phase. Figures 5 and 6 show the comparison between the profiles of the components of velocity vector, measured with PIV and predicted with CFD, in the axial and transversal direction, respectively, at times in the interval 0.160 $0.175 \mathrm{~s}$, step $0.005 \mathrm{~s}$. The magnitude of the velocity components from PIV measurements and CFD simulation shows satisfactory agreement in the first four time steps of investigation, both in the axial direction and in the transversal one. Figure 6 shows how $0.01 \mathrm{~s}$ after the systolic peak (time $0.175 \mathrm{~s}$ ), PIV measurement of the transversal velocity component and its CFD prediction do not agree satisfactorily. In Figures 7, 8 the comparison between the profiles of the components of the velocity vector, measured with PIV and predicted with CFD, in the axial and transversal direction, respectively, at times in the interval $0.180-0.190 \mathrm{~s}$, step $0.005 \mathrm{~s}$, is shown.

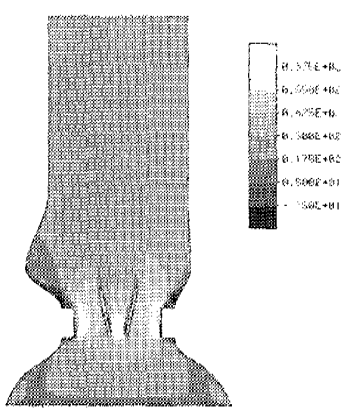

Figure 3: Map distribution for uy $(t=0.165 \mathrm{~s})$

The great difference noted between velocity profiles traced by PIV and computed with CFD after the systolic peak is due to the fact that in the deceleration phase there is a loss of organization in the flow field that becomes strongly three-dimensional; furthermore, also the geometry of the in vitro investigated model of the aortic root, with its three sinuses rather than the one axisymmetric sinus considered in previous studies [5-8], contibutes to 3D fluid dynamic behaviour. Figure 8 shows, after $0.180 \mathrm{~s}$ from the beginning of the cycle, an opposite sign in the transversal component of velocity measured by PIV with respect to the correspondent numerically simulated one. 


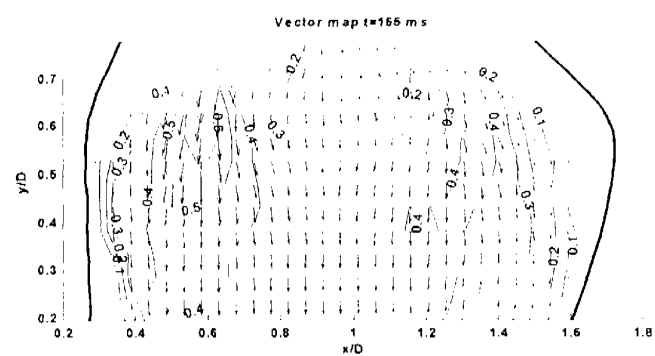

Figure 4: Velocity field from PIV investigation. The iso-velocity countor plot is presented in non-dimensional form respect to the reference velocity $(\mathrm{U}=0.626 \mathrm{~m} / \mathrm{s})$. D is the reference length $(19 \mathrm{~mm})$

This behaviour can be explained considering that the vortex in the Valsalva sinus enforces the flow in this district to be more aligned with the flow axis in the experimental set-up, while this situation is not predicted by CFD simulation.

\section{Discussion}

The hydrodynamical in vitro tests for the investigation of valves performances (as in [16]) provide only 'macroscopic' informations, being based on the measurements of quantities such as pressure drop, energy losses, regurgitation. These quantities do not describe local phenomena such as vortices development, stagnation points, flow recirculation. In vitro experiments to study the local fluid dynamics into artificial devices involve the use of advanced techniques such as LDA [17] and PIV [18]. As also observed in [4,19], these techniques could suffer from lack of spatial resolution for particular districts of the in-vitro set-up. Furthermore, these techniques disclose limitations in the analysis of transient phenomena. The above mentioned drawbacks lead to an unlikely partial lack in detailed information, with the consequence that certain 'micro' aspects of the performance of a mechanical valve on flow disturbance can not be taken into account. The intrinsic troubles in carrying out quantitative observations of local flow disturbances by any of the currently available laboratory techniques have suggested the numerical methods as a significant instrument for a better understanding of the dynamics of flowing blood.

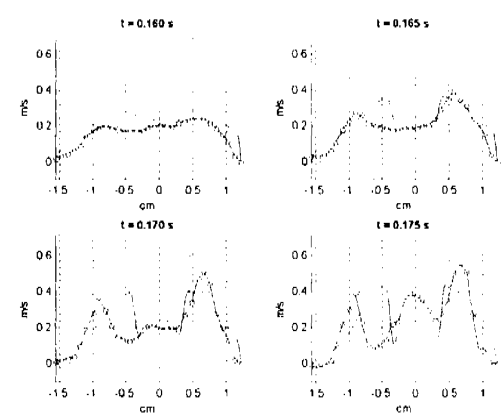

Figure 5: Axial velocity profiles (time systolic interval from 0.160 to $0.175 \mathrm{~s}$ ) (o PIV and - CFD) on the largest section of the aortic root 
The great part of the numerical models of mechanical heart valves, that are present in literature, are 2D and 3D steady and 2D time-dependent laminar flow models; only in recent years $3 \mathrm{D}$ transient models were able to predict the flow field through and downstream prosthetic valves [5-8,20], even if in simplified geometry. In $[6,9,20]$ a $3 \mathrm{D}$ model of the geometry of the ventricle, valve, sinus and aorta was developed, where the entire structure was assumed to be axisymmetric. In [9] the results of 3D, time-dependent numerical prediction of flow through a bileaflet mechanical heart valve were compared with experimental LDA measurements, with the analysis focused on the first half of systole and a peak systolic Reynolds number of 1500. The 3D time-dependent CFD model was considered to give a reasonable indication of the dominant flow patterns downstream of the bileaflet heart valve. In [19] the results of the simulation of 3D transient physiological flows in fixed geometries similar to a CarboMedics bileaflet heart valve at different opening angles, were reported, with a peak Reynolds number of 1818 , very low with respect to reality. In our simulation we investigated a fluid dynamic domain up to a Reynolds number of 3200. The comparison between computed and measured velocities provides an instrument to examine the confidence that can be placed in the study of a physical phenomenon by numerical models instead of the more complex experimental evaluation. If the numerical model reaches an adequate level of accuracy, it can bring more accurate results because there are no spatial locations where the investigation would be difficult to perform as in the experimental measurements. The study of prosthetic heart valves fluid dynamics, because of its time-dependence, interaction between fluid and valve, geometrical complexity and high velocities, needs particular care in the numerical model development. In this way many investigators preferred to study only one of the aspects said before, by neglecting their interaction or by using simpler schemes.

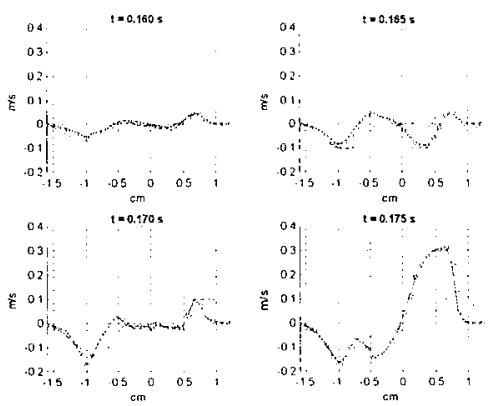

Figure 6: Transversal velocity profiles (time systolic interval from 0.160 to $0.175 \mathrm{~s}$ ) (o PIV and - CFD) on the largest section of the aortic root 


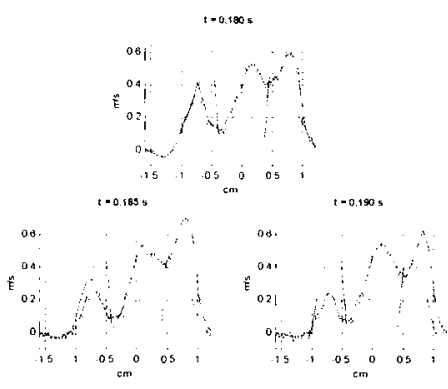

Figure 7: Axial velocity profiles (time systolic interval from 0.180 to $0.190 \mathrm{~s}$ ) (oPIV and-CFD) on the largest section of the aortic root

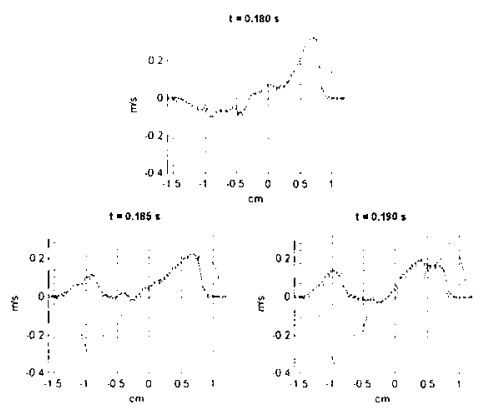

Figure 8: Transversal velocity profiles (time systolic interval from 0.180 to $0.190 \mathrm{~s}$ ) (oPIV and - CFD) on the largest section of the aortic root

In this study it was preferred to limit the time interval of the analysis to systolic peak without considering leaflets movement, but using a realistic aortic root design and the in vitro measured flow. The two methods of investigation applied in this paper differ for spatial resolution. The numerical resolution is about six times higher than PIV one: that means higher accuracy in the resolution of smaller scales of the flow and partially justifies the different location of velocities peaks reported in figures $5,6,7,8$. Figure 5 also emphasizes slight differences in the axial velocity morphologies for PIV and CFD profiles from $t=0.160$ s to $t=0.175 \mathrm{~s}$ : the reason of this difference may lie in the fluttering phenomenon starting during the faster phase of the systole, previously observed in $[6,13]$ in a CarboMedics valve. The profiles of the axial and transversal components of the velocity vector, in figures 7,8 , show a not satisfactory agreement between PIV measurements and CFD predictions after the systolic peak: in the deceleration phase this is due to a loss of organization in the flow field, that becomes strongly 3D: in this phase fluid inertial effects and turbulent mixing phenomena become significant. After $0.180 \mathrm{~s}$ from the beginning of the cycle, we observed a deviation in the left outer jet toward the centre of the aortic root in vitro but this was not predicted by CFD, as underlined by the opposite sign of the transversal component of velocity measured by PIV with respect to the correspondent numerically simulated one (figure 8 ). In our simulation no 
turbulence model was used: the favourable agreement obtained for in vitro and computed results confirms that no turbulence scheme has to be implemented for good quantitative model predictions, at least for the conditions selected in this study. The 2D time-dependent CFD model prediction of a bileaflet valve in an aortic test chamber reproducing the realistic geometry of the aortic root herein presented can be considered to give a reasonable indication of the dominant flow patterns downstream of the bileaflet heart valve in the time interval of the systole when the modeled valve can be considered as fully open and the deceleration phase has not been still generated. To get more details and complete informations about the flow field of the valve, 3D numerical calculations are needed. It must be stated that, as reported in [19], reliable simulations of 3D transient flows characterized by peak Reynolds numbers well above 1000 are still a challenge.

\section{References}

[1] Reul H, Vahlbruch A, Giersiepen M, Schmitz-Rode Th, Hirtz V, Effert S. The geometry of the aortic root in health, at valve disease and after valve replacement. Journal of Biomechanics, 23, pp. 181-91, 1990.

[2] Bellhouse BJ, Talbot C. The fluid mechanics of the aortic valve. Journal of Fluid Mechanics, 35, pp. 721-35, 1969.

[3] Moreno D, Mendoza Santoyo F, Funes-Gallanzi M, Fernandez Orozco S. An optimum velocimetry data display method. Optics \& Laser, 32, pp. 121$128,2000$.

[4] Black MM, Hose DR, Lamb CJ, Lawford PV, Ralph SJ. In vitro heart valve testing: steady versus pulsatile flow. Journal of Heart Valve Disease, 3(2), pp. 212-15, 1994.

[5] King MJ, David T, Fisher J. .An initial parametric study of fluid flow through bileaflet mechanical heart valves using computational fluid dynamics. J Eng Med, 208, pp. 63-71, 1994.

[6] King MJ, David T, Fisher J. Three-dimensional study of the effect of two leaflet opening angles on the time-dependent flow through a bileaflet mechanical heart valve. Med Eng Phys, 19(3), pp235-41, 1997.

[7] Kelly SG, Verdonck PR, Vierendeels JA, Riemslagh K, Dick E, Van Nooten GG. A three-dimensional analysis of flow in the pivot regions of an ATS bileaflet valve. Int J Artif Organs, 22(11), pp.754-63, 1999.

[8] Shipkowitz T, Ambrus J, Kurk J, Wickramasinghe K. Evaluation technique for bileaflet mechanical valves. Journal of Heart Valve Disease, 11(2), pp. 275-82, 2002.

[9] King MJ, Corden J, David T, Fisher J. .A three-dimensional, time-dependent analysis of flow through a bileaflet mechanical heart valve: comparison of experimental and numerical results. Journal of Biomechanics, 29(5), pp. 609-18, 1996.

[10] Shim EB, Chang KS. Numerical analysis of three-dimensional Bjork-Shiley valvular flow in an aorta. J Biomech Eng, $119(1)$, pp. 45-51, 1997.

[11] Lei M, van Steenhoven AA, van Campen DH. Experimental and numerical analyses of the steady flow field around an aortic Bjork-Shiley standard valve prosthesis. $J$ Biomech, 25(3), pp. 213-22, 1992. 
[12] Sutera SP, Joist JH. The haematological effects of non-physiological blood flow. In Thrombosis embolism and bleeding. Buchart EG and Bodnar E eds, ICR Publishers, London, 1992;149-159.

[13] Barbaro V., Grigioni M., Daniele C., D'Avenio G., Boccanera G. $19 \mathrm{~mm}$ sized bileaflet valve prostheses' flow field investigated by bidimensional laser Doppler anemometry (part I: velocity profiles). International Journal of Artificial Organs, 20(11), pp. 622-628, 1997.

[14] Barbaro V., Grigioni M., Daniele C., D'Avenio G. Principal stress analysis in LDA measurements of the flow field downstream of $19 \mathrm{~mm}$ Sorin Bicarbon heart valve. Technology and Health care 1998;6:259-70.

[15] Food and Drug Administration, Draft replacement heart valve guidance, 1994.

[16] Yoganathan AP, Sung HW, Woo YR, Jones M. In vitro velocity and turbulence measurements in the vicinity of three new mechanical aortic heart valve prostheses: Bjork-Shiley Monostrut, Omni-Carbon, and Duromedics. J Thorac Cardiovasc Surg, 95(5), pp. 929-39, 1988.

[17] Grigioni M, Daniele C, D'Avenio G, Barbaro V. Laser Doppler anemometry study of bidimensional flows downstream of three $19-\mathrm{mm}$ bileaflet valves in the mitral position, under kinematic similarity. Annals of Biomedical Engineering, 28(2):194-203, 2000.

[18] Lim WL, Chew Y, Chew TC, Low HT. Pulsatile flow studies of a porcine bioprosthetic aortic valve in vitro: PIV measurements and shear-induced blood damage. J Biomech, 34(11), pp. 1417-27, 2001.

[19] Krafczyk M, Cerrolaza M, Schulz M, Rank E. Analysis of 3D transient blood flow passing through an artificial aortic valve by Lattice-Boltzmann methods. Journal of Biomechanics, 31(5), pp.453-62, 1998.

[20] Cerrolaza M, Herrera M, Berrios R, Annichiaricco W. A comparison of the hydrodynamical behaviour of three heart aortic prostheses by numerical methods. J Med Eng Technol, 20(6), pp. 219-28, 1996. 